\title{
LEVANTAMENTO FLORÍSTICO EM AFLORAMENTO ROCHOSO NO PIEMONTE DA BORBOREMA, PARAÍBA, BRASIL
}

\author{
Joel Maciel Pereira Cordeiro ${ }^{1}$ \\ Bartolomeu Israel de Souza ${ }^{2}$ \\ Leonardo Pessoa Felix ${ }^{3}$
}

\begin{abstract}
Resumo: O presente estudo tem como objetivo descrever a composição florística de um afloramento rochoso localizado em Serra da Raiz, piemonte da Borborema, Paraíba, e verificar as similaridades e dissimilaridades do mesmo com outros inselbergs do Nordeste brasileiro. O trabalho registrou 138 espécies vegetais, sendo as famílias Fabaceae, Poaceae e Euphorbiaceae com maior número de espécies. Comparando este trabalho com outros estudos florísticos verifica-se que o afloramento rochoso do piemonte da Borborema compartilha mais características florísticas com as regiões circundantes, formada por matas estacionais deciduais, que com outros inselbergs do Nordeste brasileiro. Fatores relacionados à pediplanação, similaridade com áreas de entorno, distâncias geográficas e heterogeneidade dos afloramentos rochosos foram comparados e discutidos neste trabalho.
\end{abstract}

Palavras-chave: Agreste; Inselberg; Pediplanação

\section{FLORISTIC SURVEY IN OUTCROP ROCK OF PIEDMONT BORBOREMA, PARAIBA, BRAZIL}

Abstract: The aim of the present study was to describe the floristic composition of a rocky outcrop in Serra da Raiz, piedmont of Borborema, Paraíba, and verify the similarities or dissimilarities with other inselbergs northeastern Brazil. The work resulted in the registration of 138 plant species, and the families Fabaceae, Poaceae and Euphorbiaceae with the largest number of species. Comparing this work with other floristic studies, it appears that the rocky outcrop in piedmont Borborema shares floristic characteristics with the surrounding regions (seasonal forest) than with other inselbergs northeastern Brazil. Factors related to pediplanation, similarity to surrounding areas, geographical distances and heterogeneity of rocky outcrops were compared and discussed in this work.

Keywords: Agreste; Inselberg; Pediplanation

\section{LEVANTAMIENTO FLORÍSTICO EN AFLORAMIENTO ROCOSO EN EL PIEMONTE DEL BORBOREMA, PARAÍBA, BRASIL}

Resumen: El presente estudio tiene como objetivo describir la composición florística de un afloramiento rocoso localizado en la municipalidad de Serra da Raiz/Paraíba, piemonte de

\footnotetext{
${ }^{1}$ Aluno do curso de Doutorado em Agronomia pela Universidade Federal da Paraíba. Email: joelmpcordeiro@yahoo.com.br

${ }^{2}$ Professor do Departamento de Geociências do Centro de Ciências Exatas e da Natureza, Universidade Federal da Paraíba. Email: bartolomeuisrael@gmail.com

${ }^{3}$ Professor do Departamento de Biologia do Centro de Ciências Agrárias, Universidade Federal da Paraíba. Email: lpfelix2@gmail.com
} 
Borborema, y verificar las semejanzas y disimilaridades del mismo con otros inselbergs del Nordeste brasileño. El trabajo registró 138 especies vegetales, siendo las familias Fabaceae, Poaceae y Euphorbiaceae con mayor número de especies. Comparando este trabajo con otros estudios florísticos se verifica que el afloramiento rocoso del piemonte de Borborema comparte más características florísticas con las regiones circundantes, formada por bosques estacionales deciduales, que con otros inselbergs del Nordeste brasileño. Los factores relacionados con la pediplanación, la similitud con áreas de entorno, distancias geográficas y heterogeneidad de los afloramientos rocosos fueron comparados y discutidos en este trabajo.

Palabras clave: Agreste; Inselberg; Pediplanación

\section{INTRODUÇÃO}

No Nordeste brasileiro o piemonte da Borborema corresponde a uma formação geomorfológica caracterizada por um conjunto de serras (esporões) que se estende no sentido norte-sul entre a Depressão Sublitorânea e a encosta oriental do planalto da Borborema (CARVALHO, 1982). Esta região apresenta um soerguimento de aproximadamente 400 metros em relação a suas áreas vizinhas, o que propicia uma maior umidade favorecida pelos ventos alísios de sudeste (FELICIANO; MELO, 2003).

No piemonte da Borborema a geologia corresponde a um complexo gnáissicogranítico-migmatítico (CARVALHO, 1982) e as feições nos topos de serras propiciam a formação de diversos afloramentos rochosos que emergem através do processo erosivo do solo ao longo de milhares de anos.

Diferente dos inselbergs encontrados na Depressão Sertaneja e no Agreste da Borborema, formados em meio às superfícies aplainadas (PORTO et al., 2008; LUCENA et al., 2015), os afloramentos rochosos do piemonte da Borborema se apresentam em meio a superfícies acidentadas formadas por serras. Entretanto, diversas características como temperaturas mais altas que as áreas do entorno, baixa umidade, maior exposição a ventos, retenção de calor e maior escoamento de água são elementos comuns neste tipo de ambiente (POREMBSKI, 2007).

Floristicamente, os inselbergs são caracterizados por abrigar uma vegetação formada por plantas xerófitas e suculentas, sendo estas dotadas de alterações morfológicas e fisiológicas (sistema radicular, tricomas, estômatos, etc.) que as possibilitam sobreviverem ao estresse ambiental nestas áreas (POREMBSKI, 2007). Entre as espécies vegetais comumente encontradas nos inselbergs do Nordeste brasileiro destacam-se Encholirium spectabile Mart. ex Schult. \& Schult. f. (Bromeliaceae), Mandevilla tenuifolia (Mikan) R. E. Woodson. (Apocynaceae), Cnidoscolus urens (L.) Arthur, Senna martiana (Benth.) H.S.irwin \& 
Barneby, Cissus simsiana Schult. \& Schult.f., e inúmeras espécies pertences especialmente as famílias Fabaceae, Asteraceae, Poceae e Cyperaceae (PORTO et al., 2008; GOMES; ALVES, 2009, 2010; LUCENA et al., 2015).

Embora os estudos florísticos dos inselbergs no Nordeste brasileiro tenham aumentado nas últimas décadas, especialmente referentes a determinadas áreas da Bahia (FRANÇA et al., 1997; CONCEIÇÃO; PIRANI, 2007), assim como no Agreste da superfície aplainada do planalto da Borborema (PORTO et al., 2008; GOMES; ALVES, 2009; SALES-RODRIGUES et al., 2014) e baixo sertão (LUCENA et al., 2015), verifica-se, contudo, que pouco se sabe sobre a flora nas serras que correspondem ao piemonte da Borborema, especialmente em relação aos afloramentos rochosos que ocorrem na mesma.

Nesta concepção, o referente trabalho tem como objetivo caracterizar a diversidade florística em um afloramento rochoso localizado no piemonte da Borborema, município de Serra da Raiz, Paraíba. Com os resultados do trabalho pretende-se responder as seguintes questões: a) qual a composição florística deste afloramento rochoso? b) os afloramentos rochosos do piemonte da Borborema se assemelham floristicamente a outros inselbergs do Nordeste brasileiro? c) As espécies do afloramento rochoso no piemonte da Borborema compartilham características com a vegetação do Agreste paraibano, ou formam grupos vegetais próximos a outros inselbergs?

\section{MATERIAL E MÉTODOS}

Área de Estudo

O referente trabalho foi realizado em um afloramento rochoso localizado no Distrito de Cardoso, Município de Serra da Raiz, Paraíba, região Nordeste do Brasil (06 $40^{\prime} 44^{\prime \prime}$; $\left.35^{\circ} 26^{\prime} 23^{\prime \prime} \mathrm{W}\right)$. A área corresponde a um afloramento rochoso granítico de aproximadamente 2ha, com até $20 \mathrm{~m}$ de diferença entre a altitude da base e o topo (Figura 1).

O clima da região é do tipo As' quente e úmido, com chuvas de outono-inverno, temperatura média de $26^{\circ} \mathrm{C}$ e precipitação média de $900 \mathrm{~mm}$ por ano (FELICIANO; MELO, 2003). O relevo pertence ao piemonte da Borborema, caracterizado por um conjunto de serras (esporões), com altitude em torno de 350m em relação ao nível do mar (CARVALHO, 1982).

A cobertura vegetal pertence ao Agreste Sublitorâneo, composta por vegetação do tipo Floresta Estacional Decidual, com a maioria das espécies caducifólias e espinhosas, fazendose presentes espécies da Caatinga e da Mata Atlântica, esta última com menor representatividade (IBGE, 2012; CORDEIRO et al., 2015). 


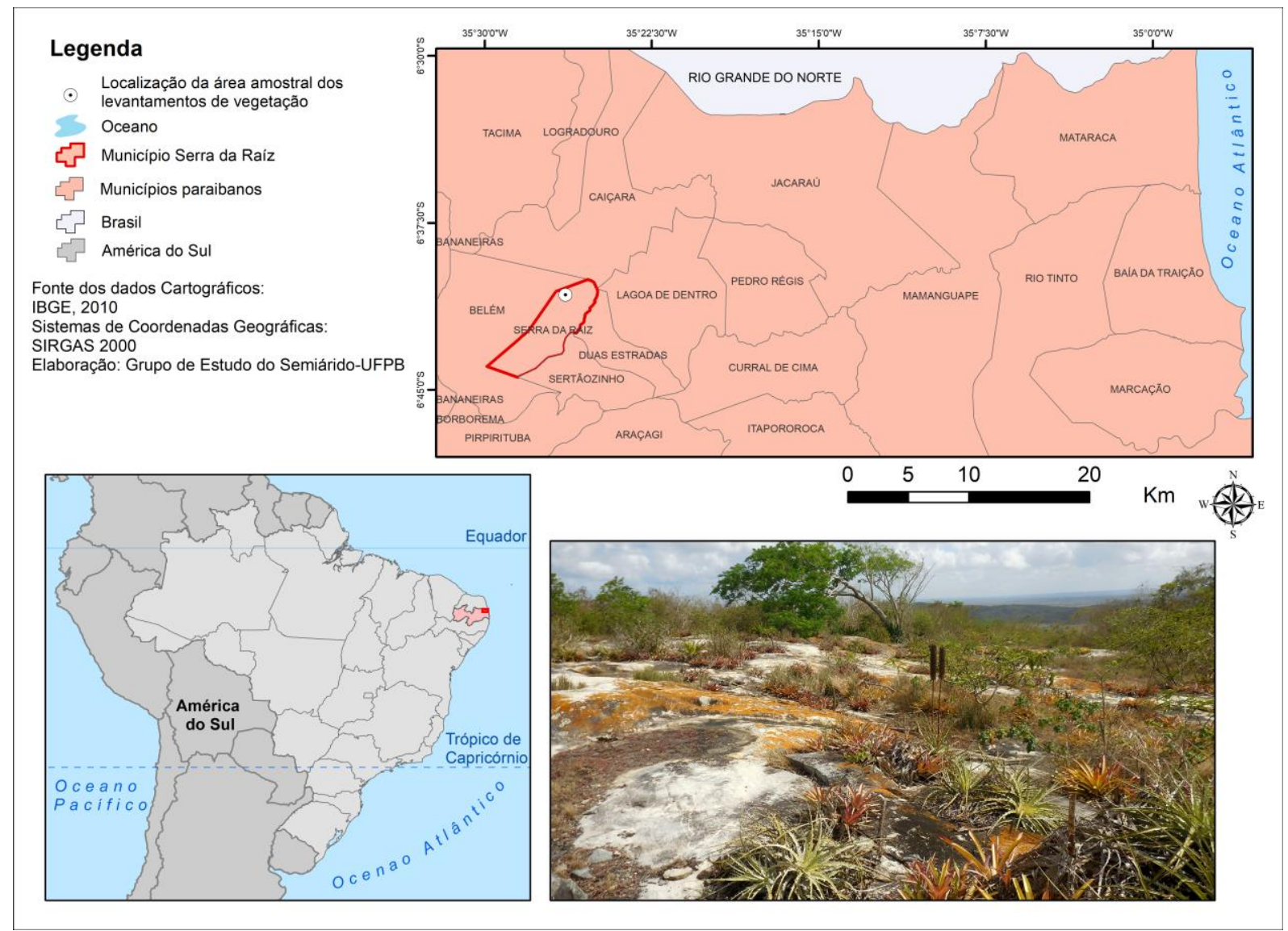

Figura 01 - Localização geográfica do afloramento rochoso analisado, Serra da Raiz, Paraíba, região Nordeste do Brasil.

Levantamento Florístico

Para o registro florístico foram realizadas coletas de material botânico no inselberg entre junho de 2014 e fevereiro de 2016, incluindo períodos secos e chuvosos. O levantamento foi desenvolvido seguindo os procedimentos adaptados ao bioma Caatinga conforme Rodal et al. (1992), onde se é produzido uma lista florística completa com o nome das espécies presentes na área de estudo, incluindo árvores, arbustos, ervas, epífitas e lianas.

As espécies em idade fértil foram fotografadas, coletadas e enviadas ao herbário Professor Jayme Coelho de Moraes (EAN) da Universidade Federal da Paraíba. A identificação das espécies foi estabelecida ainda em campo e, quando a mesma não foi possível de ser realizada, recorreu-se a bibliografia especializada, morfologia comparada com exsicatas depositadas no herbário EAN e envio a especialistas. As espécies foram organizadas em suas respectivas famílias botânicas conforme a mais recente classificação das plantas superiores, APG IV (ANGIOSPERM PHYLOGENY GROUP, 2016). 


\section{RESULTADOS E DISCUSSÃO}

Foram registradas no afloramento rochoso localizado em Serra da Raiz, piemonte da Borborema, um total de 138 espécies vegetais (Figura 02, Tabela 01). Entre as espécies registradas, nove foram identificadas ao nível de gênero, duas permaneceram identificadas apenas ao nível de família, e uma espécie de arvoreta não foi identificada por falta de material botânico reprodutivo.

As famílias mais representativas foram Fabaceae com 20 espécies, seguida de Poaceae com nove espécies, Euphorbiaceae e Asteraceae com oito espécies, Cyperaceae (sete espécies) e Orchidaceae, Bignoniaceae e Bromeliaceae com cinco espécies cada, totalizando 49,63\% das espécies registradas. Os gêneros com maiores números de espécies foram Senna Mill. (quatro), seguido de Mimosa L. e Portulaca L. com três espécies cada.

O levantamento florístico realizado em afloramento rochoso do piemonte da Borborema, localizado em Serra da Raiz, Paraíba, revelou determinadas semelhanças com outros levantamentos realizados em inselbergs do Nordeste brasileiro, seja em relação às principais famílias botânicas ou a determinadas espécies registradas no mesmo.

As famílias botânicas com maior número de espécies registradas neste estudo (Fabaceae, Poaceae, Euphorbiaceae, Asteraceae, Cyperaceae, Orchidaceae e Bromeliaceae) aparecem comumente mencionadas como as mais diversas também em muitos outros levantamentos florísticos realizados neste tipo de ambiente (POREMBSKI et al., 1997; ARAÚJO et al., 2008; GOMES; ALVES, 2009, 2010; LUCENA et al., 2015). Nas regiões Neotropicais, especialmente no Brasil, as famílias botânicas acima mencionadas aparecem com as maiores quantidade e diversidade de espécies, principalmente nos domínios da Caatinga e Mata Atlântica (SOUZA; LORENZI, 2012; JUDD et al., 2009; FORZZA et al., 2010). 

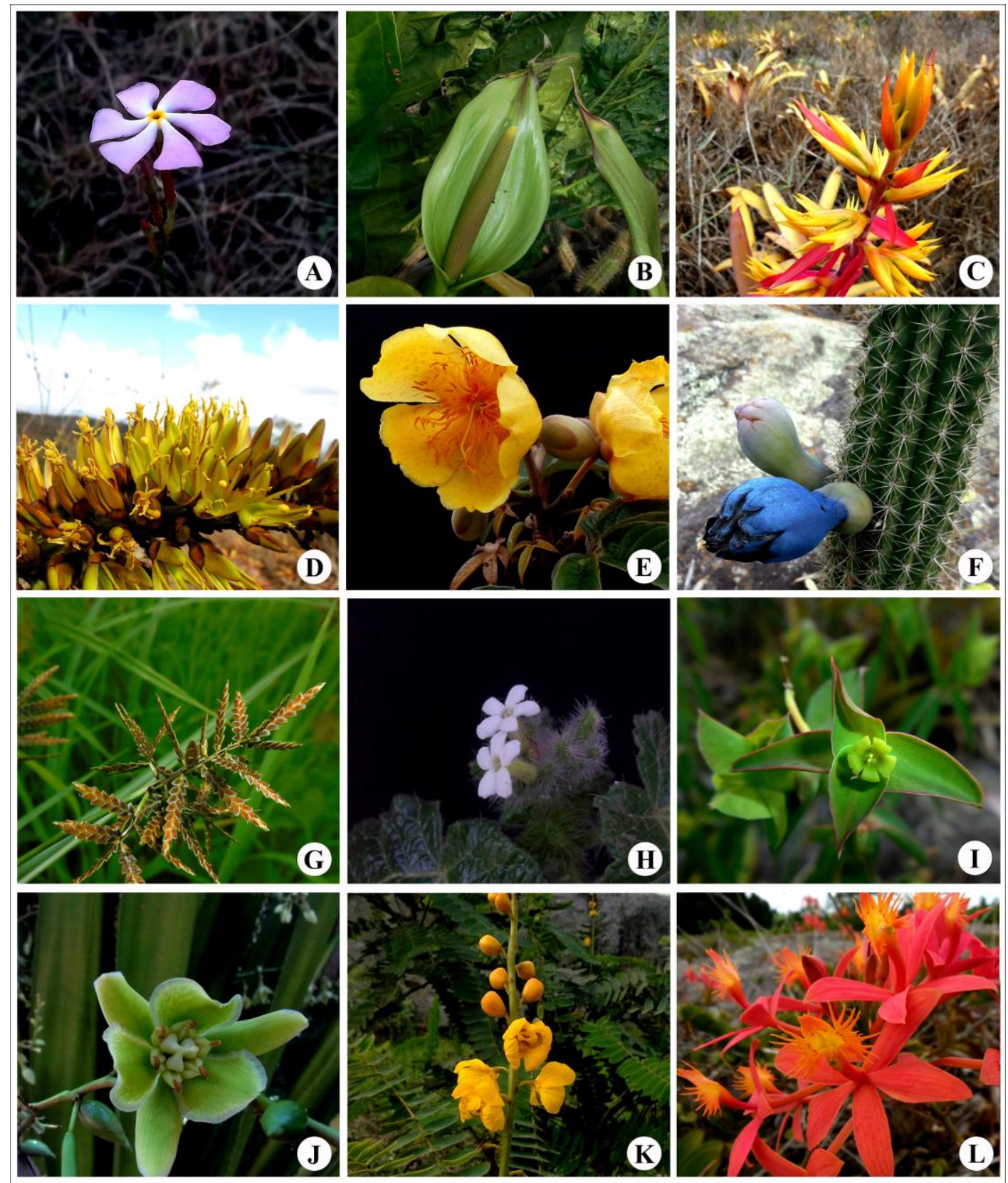

Figura 02 - Principais espécies vegetais encontradas no afloramento rochoso, Serra da Raiz, Paraíba: A. Mandevilla tenuifolia, B. Anthurium affine, C. Aechmea aquilega, D. Encholirium spectabile, E. Cochlospermum vitifolium, F. Pilosocereus pachycladus, G. Cyperus compressus, H. Cnidoscolus urens, I. Euphorbia comosa, J. Furcraea foetida, K. Senna martiana, L. Epidendrum cinnabarinum.

Tabela 01 - Lista de espécies vegetais registradas em afloramento rochoso localizado no município de Serra da Raiz, piemonte da Borborema, Paraíba, Brasil.

\begin{tabular}{ll}
\hline Família & Espécie \\
\hline Acanthaceae & Ruellia bahiensis (Nees) Morong. \\
\hline
\end{tabular}


Amaryllidaceae

Anacardiaceae

Apocynaceae

Araceae

Asparagaceae

Asteraceae

Bignoniaceae

Bixaceae

Boraginaceae

Bromeliaceae
Hippeastrum stylosum Herb.

Spondias mobin L.

Allamanda blanchetii A. DC.

Mandevilla tenuifolia (Mikan) R.E.Woodson

Matelea ganglinosa (Vell.) Rapini

Anthurium affine Schott

Lemna valdiviana Phil.

Philodendron acutatum Schott

Wolffia brasiliensis Wedd.

Agave sisalana Perr.

Furcraea foetida (L.) Haw.

Acmella sp.

Bidens pilosa $\mathrm{L}$.

Conocliniopsis prasiifolia (DC.) R.M.King \& H.Rob.

Conyza bonariensis (L.) Cronquist

Eclipta prostrata (L.) L.

Tridax procumbens $\mathrm{L}$.

Vernonanthura brasiliana (L.) H.Rob.

Indet.

Dolichandra quadrivalvis (Jacq.) L.G.Lohmann

Fridericia pubescens (L.) L.G.Lohmann

Handroanthus impetiginosus (Mart. ex DC.) Mattos

Jacaranda jasminoides (Thunb.) Sandwith

Tabebuia roseoalba (Ridl.) Sandwith

Cochlospermum vitifolium (Willd.) Spreng.

Myriopus rubicundus (Salzm. ex DC.) Luebert

Varronia dardani (Taroda) J.S.Mill.

Aechmea aquilega (Salisb.) Griseb.

Bromelia karatas L.

Encholirium spectabile Mart. ex Schult. \& Schult.f.

Hohenbergia catingae Ule

Tillandsia recurvata (L.) L.

Burseraceae

Commiphora leptophloeos (Mart.) J.B.Gillett 


\begin{tabular}{|c|c|}
\hline \multirow[t]{2}{*}{ Cactaceae } & Cereus jamacaru DC. \\
\hline & Pilosocereus pachycladus F.Ritter \\
\hline \multirow[t]{2}{*}{ Celastraceae } & Hippocratea volubilis L. \\
\hline & Pristimera celastroides (Kunth) A.C.Sm. \\
\hline Cleomaceae & Hemiscola diffusa (Banks ex DC.) Iltis \\
\hline \multirow[t]{2}{*}{ Combretaceae } & Combretum fruticosum (Loefl.) Stuntz \\
\hline & Combretum leprosum Mart. \\
\hline Commelinaceae & Commelina obliqua Vahl. \\
\hline \multirow[t]{3}{*}{ Convolvulaceae } & Ipomoea brasiliana (Choisy) Meisn. \\
\hline & Ipomoea setosa Ker Gawl. \\
\hline & Merremia aegyptia (L.) Urb. \\
\hline \multirow[t]{7}{*}{ Cyperaceae } & Cyperus compressus L. \\
\hline & Cyperus surinamensis Rottb. \\
\hline & Kyllinga odorata Vahl \\
\hline & Pycreus sp. \\
\hline & Rhynchospora barbata (Vahl) Kunth \\
\hline & Rhynchospora globosa (Kunth) Roem. \& Schult. \\
\hline & Scleria sp. \\
\hline Erythroxylaceae & Erythroxylum caatingae Plowman \\
\hline \multirow[t]{8}{*}{ Euphorbiaceae } & Cnidoscolus urens (L.) Arthur \\
\hline & Croton urticifolius L. \\
\hline & Croton glandulosus L. \\
\hline & Dalechampia scandens L. \\
\hline & Euphorbia comosa Vell. \\
\hline & Euphorbia hyssopifolia L. \\
\hline & Manihot glaziovii Müll. Arg. \\
\hline & Sapium argutum (Müll. Arg.) Huber. \\
\hline \multirow[t]{6}{*}{ Fabaceae } & Aeschynomene sensitiva $\mathrm{Sw}$. \\
\hline & Bauhinia cheilantha (Bong.) Steud. \\
\hline & Centrosema brasilianum (L.) Benth. \\
\hline & Chamaecrista nictitans (L.) Moench \\
\hline & Dioclea violacea Mart. ex Benth. \\
\hline & Enterolobium contortisiliquum (Vell.) Morong. \\
\hline
\end{tabular}


Libidibia ferrea (Mart. ex Tul.) L.P.Queiroz

Machaerium hirtum (Vell.) Stellfeld

Mimosa hirsutissima Mart.

Mimosa arenosa (Willd.) Poir.

Mimosa sensitiva $\mathrm{L}$.

Piptadenia stipulacea (Benth.) Ducke

Poincianella pyramidalys (Tul.) L.P.Queiroz

Senegalia tenuifolia (L.) Britton \& Rose

Senna georgica H.S.Irwin \& Barneby

Senna macranthera (DC. ex Collad.) H.S.Irwin \& Barneby

Senna martiana (Benth.) H.S.Irwin \& Barneby

Senna obtusifolia (L.) H.S.Irwin \& Barneby

Zornia myriadena Benth.

Lamiaceae

Marsypianthes chamaedrys (Vahl) Kuntze

Ocimum campechianum Mill.

Vitex rufescens A.Juss.

Loasaceae

Aosa rupestris (Gardner) Weigend

Loganiaceae

Spigelia anthelmia L.

Strychnos parvifolia A. DC.

Loranthaceae

Passovia pyrifolia (Kunth) Tiegh.

Malpighiaceae

Diplopterys lutea (Griseb.) W.R.Anderson \& C.C.Davis

Stigmaphyllon paralias A. Juss.

Malvaceae

Ceiba glaziovii (Kuntze) K.Schum.

Herissantia crispa (L.) Brizicky

Pavonia cancellata (L.) Cav.

Sidastrum paniculatum (L.) Fryxell

Marantaceae

Maranta zingiberina L.Andersson

Melastomataceae

Comolia villosa (Aubl.) Triana

Tibouchina grandifolia Cogn.

Myrtaceae

Campomanesia aromatica (Aubl.) Griseb.

Eugenia sp.

Psidium guineense $\mathrm{Sw}$.

Onagraceae

Ludwigia octovalvis (Jacq.) P.H.Raven 
Orchidaceae

Oxalidaceae

Passifloraceae

Poaceae

Polygalaceae

Portulacaceae

Rhamnaceae

Rubiaceae

Salicaceceae

Sapindaceae

Simaroumbaceae

Smilacaceae

Solanaceae
Cyrtopodium flavum Link \& Otto ex Rchb.f.

Epidendrum cinnabarinum Salzm

Oeceoclades maculata (Lindl.) Lindl.

Prescottia plantaginifolia Lindl. ex Hook.

Sacoila lanceolata (Aubl.) Garay

Oxalis cratensis Oliv. ex Hook

Passiflora cincinnata Mart.

Passiflora foetida $\mathrm{L}$.

Andropogon sp.

Aristida adscensionis L.

Cenchrus echinatus L.

Melinis minutiflora P.Beauv.

Setaria parviflora (Poir.) Kerguélen

Setaria sp.

Sporobolus sp.

Urochloa decumbens (Stapf) R.D.Webster

Indet.

Securidaca diversifolia (L.) S.F.Blake

Portulaca halimoides L.

Portulaca oleracea L.

Portulaca sp.

Gouania colurnifolia Reissek

Ziziphus joazeiro Mart.

Borreria verticillata (L.) G.Mey.

Coutarea hexandra (Jacq.) K.Shum.

Diodella teres (Walter) Small

Richardia grandiflora (Cham. \& Schltdl.) Steud.

Prockia crucis P.Browne ex L.

Paullinia pinnata L.

Serjania sp.

Simaba floribunda A.St.-Hil.

Smilax brasiliensis Spreng.

Solanum rhytidoandrum Sendtn. 


\begin{tabular}{ll}
\hline Turneraceae & Turnera subulata $\mathrm{Sm}$. \\
Verbenaceae & Lantana camara $\mathrm{L}$. \\
& Lantana canescens Kunth. \\
Vitaceae & Cissus simsiana Schult. \& Schult.f. \\
Indet. & Indet. \\
\hline
\end{tabular}

As espécies Mandevilla tenuifolia (Mikan) R.E. Woodson, Anthurium affine Schott, E. spectabile, C. urens, S. martiana, Tibouchina grandifolia Cogn., Cyrtopodium flavum Link \& Otto ex Rchb.f. e Cissus simsiana Schult. \& Schult.f., registradas neste afloramento rochoso, são tipicamente encontradas em inselbergs (ARAÚJO et al., 2008; GOMES; ALVES, 2009, 2010; SALES-RODRIGUES et al., 2014; LUCENA et al., 2015), demonstrando que os afloramentos rochosos do piemonte da Borborema e os inselbergs de várias regiões do Nordeste brasileiro compartilham muitas características florísticas em comum.

Em contrapartida, verifica-se que das 138 espécies registradas no afloramento rochoso, 64 delas $(46,37 \%)$ podem ser encontradas em fragmentos de floresta estacional decidual no Agreste sublitorâneo (CORDEIRO, FÉLIX, 2014; CORDEIRO et al., 2015), região a qual o piemonte da Borborema faz parte (FELICIANO; MELO, 2003).

Uma hipótese para explicar as características florísticas neste afloramento rochoso, onde ocorrem mais espécies em comum com a vegetação circundante que em comparação com outros inselbergs, seria o processo de pediplanação. No Nordeste brasileiro, as áreas de serras, como ocorrem no piemonte da Borborema, podem indicar um estágio intermediário do processo de pediplanação (AB'SÁBER, 1974; PRADO, 2003), onde os afloramentos rochosos estão ainda em processo inicial de soerguimento, apresentando menores desníveis altimétricos em relação às áreas vizinhas, menor declividade e, por conseguinte, compartilhando ainda diversas características florísticas com as áreas do entorno. Os inselbergs, especialmente na depressão sertaneja, são os descendentes mais antigos no processo de pediplanação (AB'SÁBER, 1974; PRADO, 2003), e apresentam maiores declividade e desnível altimétrico em relação ás áreas vizinhas e, consequentemente, exibem composição florística mais isolada em relação à vegetação do entorno.

Outra hipótese seria que a similaridade florística entre os inselbergs e a vegetação das áreas circundantes constituiria um fenômeno correlacionado, porém ainda pouco discutido na literatura. Muitos autores preferem enfatizar as discussões de similaridade florística existente entre diferentes inselbergs (CONCEIÇÃO; PIRANI, 2007; PORTO et al., 2008; GOMES; ALVES, 2009, 2010), entretanto, em alguns estudos, estes afloramentos rochosos podem ser 
mais similares com as formações vegetais circundantes que entre inselbergs de diferentes regiões. Araújo et al. (2008), por exemplo, verificou que 13\% das espécies registradas em um inselberg no Ceará foram comuns com a área de entorno, enquanto apenas 5\% das espécies foram observadas em outros inselbergs. Entretanto, necessita-se de estudos comparativos mais aprofundados entre inselbergs e vegetação circundante em diferentes regiões para confirmar esta hipótese.

Embora a distância geográfica também seja apontada como um dos principais fatores de diferenças florísticas entre inselbergs (ARAÚJO et al., 2008), verifica-se que, mesmo em áreas próximas, estes afloramentos rochosos apresentam-se bastantes diferentes enquanto sua composição florística. Comparando as espécies registradas neste trabalho com levantamentos realizados no Agreste da Borborema na Paraíba, por exemplo, observa-se a ocorrência comum de apenas 27 espécies (10,3\%) em Porto et al. (2008) e 16 espécies (7,13\%) em SalesRodrigues et al. (2014).

Ainda que seja em inselbergs de uma mesma localidade, a similaridade florística apresenta-se relativamente baixa, conforme se observa em Tölke et al. (2011) e SalesRodrigues et al. (2014). Estes autores estudaram dois diferentes inselbergs no mesmo município (Puxinanã, Paraíba) e registraram 174 espécies em ambos, das quais, apenas 20 $(11,49 \%)$ foram comuns entre estes inselbergs.

Uma vez que a distância geográfica não explica precisamente a dessemelhança florística entre inselbergs, outros elementos podem ser apontados, especialmente relacionados a fatores abióticos, como substrato e heterogeneidade do afloramento rochoso (POREMBSKI et al., 1997). Entre estes elementos que favorecem a heterogeneidade dos inselbergs podem ser citados:

I - Área (ha) do afloramento rochoso - inselbergs com áreas mais amplas apresentam um maior número de espécies (comparar, por exemplo, CONCEIÇÃO; PIRANI, 2007; GOMES; ALVES, 2009; TÖLKE et al., 2011);

II - A diferença de altitude entre a base e o topo - áreas com maiores desníveis altimétricos tendem a apresentar maior riqueza de espécies devido à formação de diferentes fitofisionomias na base, meia encosta e topo (ver, por exemplo, FRANÇA et al., 1997);

III - Declividade - áreas muito íngremes geralmente apresentam apenas rochas expostas, com baixa ocorrência de espécies vegetais, enquanto áreas mais aplainadas tendem a facilitar o acúmulo de matéria orgânica e formação de solos, contribuindo para o 
surgimento, inclusive de espécies arbóreas (ver, por exemplo, MEIRELLES et al., 1999; CONCEIÇÃO; PIRANI, 2007);

IV - geomorfologia e continentalidade - afloramentos rochosos localizados em maiores altitudes e/ou próximos ao oceano recebem, consequentemente, maior umidade, o que favorece a diversidade de espécies, com ocorrências, inclusive, de Orquidáceas e Bromeliáceas epífitas (comparar, por exemplo, GOMES; ALVES, 2009; LUCENA et al., 2015).

Desta forma, diferentes inselbergs que têm o maior número destas características em comum possivelmente apresentam também maior similaridade florística, enquanto aqueles que compartilham poucas destas características entre si consequentemente apresentariam maior heterogeneidade florística. Estas considerações poderiam explicar a similaridade mais elevada ente inselbergs nos trabalhos de França et al. (1997) e Gomes e Alves (2010), onde ambos realizaram levantamentos florísticos distintos em dois diferentes inselbergs e registraram $30,8 \%$ e $41,3 \%$ de similaridade, respectivamente.

De qualquer forma, os inselbergs formam um grupo especial de região ecológica, abrigando grande número de espécies endêmicas, ou mesmo espécies que ainda não foram descritas na literatura (SALES-RODRIGUES et al., 2014), com grandes adaptações aos rigores climáticos existentes no mesmo. Maiores amostragens entre diferentes inselbergs, o desenvolvimento de análises comparativas com a vegetação circundante ou entre inselbergs de diferentes fisionomias poderiam explicar mais precisamente a similaridade ou dissimilaridade existentes entre os afloramentos rochosos das regiões Neotropicais.

\section{Considerações Finais}

O estudo florístico desenvolvido em afloramento rochoso do piemonte da Borborema, Paraíba, região Nordeste no Brasil revelou notável diversidade florística (138 espécies). Diversas características observadas no mesmo, como as famílias mais representativas (Fabaceae, Poaceae, Asteraceae, Cyperaceae) e o registro das espécies Encholirium spectabile, Mandevilla tenuifolia, Cnidoscolus urens, Pilosocereus pachycladus, Senna martiana, Tibouchina grandifolia e Cissus simsiana são as particularidades compartilhadas entre o afloramento rochoso do piemonte da Borborema e outros inselbergs do Nordeste brasileiro.

Em contrapartida, observa-se que a composição florística deste afloramento rochoso compartilha maior similaridade com a vegetação circundante (matas secas estacionais do agreste paraibano) quando comparados com outros inselbergs do Nordeste brasileiro. 
Diversos fatores podem justificar esta dissimilaridade florística entre inselbergs, como variações no processo de pediplanação, distâncias geográficas, declividade, geomorfologia, continentalidade e, principalmente, a heterogeneidade existente entre inselbergs.

\section{Referencias Bibliográficas}

AB'SÁBER, A. N. O domínio morfoclimático semi-árido das Caatingas brasileiras. Geomorfologia, v.43, p.1-39, 1974.

ANGIOSPERM PHYLOGENY GROUP. An update of the Angiosperm Phylogeny Group classification for the order and families of flowering plants: APG IV. Botanical Journal of the Linnean Society, v.181, p.1-20, 2016.

ARAÚJO, F. S.; OLIVEIRA, R. F.; LIMA-VERDE, L. W. Composição, espectro biológico e síndrome de dispersão da vegetação de um inselberg no domínio da Caatinga, Ceará. Rodriguésia, v.59, n.4, p.659-671, 2008.

CARVAlHO, M. G. Classificação geomorfológica do estado da Paraíba. João Pessoa: Universitária/Funap, 1982.

CONCEIÇÃO, A. A.; PIRANI, J. R. Diversidade em quatro áreas de campos rupestres na Chapada Diamantina, Bahia, Brasil: espécies distintas, mas riqueza similares. Rodriguésia, v.58, n.1, p.193-206, 2007.

CORDEIRO, J. M. P. et al. Levantamento florístico preliminar da caatinga sublitorânea na Paraíba, Nordeste do Brasil. Geografia, Rio Claro, v.40, n.2, p.241-257, 2015.

CORDEIRO, J. M. P.; FELIX, L. P. Levantamento fitossociológico em mata de encosta no Agreste paraibano. Geoambiente on-line, v.21, p.13-28, 2013.

FELICIANO, M. L. M.; MELO, R. B. Atlas do Estado da Paraíba: informações para gestão do patrimônio natural. SEPLAN/IDEME/APAN/UFPB, João Pessoa, 2003. FORZZA, R. C. et al. As Angiospermas do Brasil. In: FORZZA, R. C. et al. (ed.). Catálogo de plantas e fungos do Brasil. Rio de Janeiro: Andrea Jakobsson Estúdio, 2010. p.78-89.

FRANÇA, F.; MELO, E.; SANTOS, C. C. Flora de inselbergs da região de Milagres, Bahia, Brasil: caracterização da vegetação e lista de espécies de dois inselbergs. Sitientibus Série Ciências Biológicas, v.17, p.163-184, 1997.

GOMES, P.; ALVES, M. Floristic and vegetational aspects of on inselberg in the semi-arid region of Northeast Brazil. Edinburgh Journal of Botany, v.66, n.2, p.329-346, 2009.

GOMES, P.; ALVES, M. Floristic diversity of two crystalline rocky outcrops in the Brazilian northeast semi-arid region. Revista Brasileira de Botânica, v.33, n.4, p.661-676, 2010.

INSTITUTO BRASILEIRO DE GEOGRAFIA E ESTATÍSTICA. Manual Técnico da Vegetação Brasileira. IBGE, Rio de Janeiro, 2012.

JUDD, W. S. et al. Sistemática vegetal um enfoque filogenético. Artmed: Porto Alegre, 2009.

LUCENA, D. S. et al. Flora vascular de um inselberg na mesorregião do sertão paraibano, nordeste do Brasil. Scientia Plena, v.11, n.1, p.1-11, 2015.

MEIRELLES, S. T.; PIVELLO, V. R.; JOLY, C. A. The vegetation of granite rock outcrops in Rio de Janeiro, Brazil, and the need for its protection. Environmental Conservation, v.26, n.1, p.10-20, 1999.

POREMBSKI, S. Tropical inselbergs: habitat types, adaptive strategies and diversity patterns. Revista Brasileira de Botânica, v.30, n.4, p.579-586, 2007.

POREMBSKI, S.; SEINE, R.; BARTHLOTT, W. Inselberg vegetation and biodiversity of granite outcrops. Journal of the Royal Society of Western Australia, v.80, p.193-199, 1997. 
PORTO, P. A. F et al. Composição florística de um inselberg no agreste paraibano, município de Esperança, Nordeste do Brasil. Caatinga, v.21, n.2, p.214-223, 2008.

PRADO, D. E. As caatingas da América do Sul. In: LEAL, I.R.; TABARELLI, M.; SILVA, J.M.C. (Ed.). Ecologia e conservação da caatinga. Editora Universitária da UFPE, Recife, 2003. p.3-73.

RODAL, M. J. N.; SAMPAIO, E. V. S. B.; FIGUEIREDO, M. A. Manual sobre métodos de estudo florístico e fitossociológico - ecossistema Caatinga. Sociedade Botânica do Brasil, Brasília, 1992.

SALES-RODRIGUES, J.; BRASILEIRO, J. C.; MELO, J. I. M. Flora de um inselberg na mesorregião agreste do estado da Paraíba-Brasil. Polibotánica, v.37, p.47-61, 2014.

SOUZA, V. C.; LORENZI, H. Botânica sistemática: guia ilustrado para identificação das famílias de Fanerógamas nativas e exóticas no Brasil, baseado em APG III. - 3. ed. Nova Odessa, SP: Instituto Plantarum, 2012.

TÖLKE, E. E. A. D. et al. Flora vascular de um inselberg no estado da Paraíba, Nordeste do Brasil. Biotemas, v.24, n.4, p.39-48, 2011.

Recebido em 08 de novembro de 2016.

Aceito em 19 de março de 2018. 